\title{
Propuesta de Innovación Didáctica con Portafolios Electrónicos en Formato Blog: Un Caso de Estudio en una Universidad
}

\author{
David Cobos-Sanchiz $^{(1) \star}$, Eloy López-Meneses ${ }^{(1)}$ y Mercedes Llorent-Vaquero ${ }^{(2)}$ \\ (1) Universidad Pablo de Olavide, Facultad de Ciencias Sociales. Carretera de Utrera, km. 1. 41013, \\ Sevilla-España (e-mail: dcobos@upo.es; elopmen@upo.es) \\ (2) Universidad de Sevilla, Facultad de Ciencias de la Educación. Calle Pirotecnia, s/n. 41013, \\ Sevilla-España (e-mail: mllorent@us.es) \\ ${ }^{*}$ Autor a quien debe ser dirigida la correspondencia
}

Recibido Mar. 18, 2016; Aceptado May. 2, 2016; Versión final Jun. 30, 2016, Publicado Dic. 2016

\begin{abstract}
Resumen
Este artículo presenta una experiencia de innovación docente universitaria con portafolios electrónicos del estudiantado, construidos en formato blog. El objetivo general de la investigación se concreta en el análisis de los blogs de los estudiantes en base a tres dimensiones: aspectos técnicos, aspectos educativos y aspectos sociales. La experiencia se ha llevado a cabo con 86 estudiantes del Grado de Educación Social y Doble Grado de Educación Social y Trabajo Social de la Universidad Pablo de Olavide, de Sevilla (España). El análisis de los datos se realiza mediante un proceso de codificación con un sistema categorial elaborado ad hoc y con ayuda del programa informático Atlas ti. Los resultados evidencian, entre otras cuestiones, la utilidad del portafolio electrónico para el desarrollo de nuevas habilidades y destrezas, con relación a las competencias profesionales que el estudiante ha de adquirir.
\end{abstract}

\section{Proposal for Didactic Innovation with Electronic Portfolios in Blog Format: A Case Study at a University}

\begin{abstract}
This article presents an experience of university teaching innovation with student electronic portfolios built in blog format. The overall objective of the research is to analyze student blogs according to three dimensions: technical, educational and social aspects. The experience was carried out with 86 students of the Degree of Social Education and Double Degree of Social Education and Social Work at the University Pablo de Olavide, Seville (Spain). The data analysis is performed by an encoding process with a categorical system prepared ad hoc and using the Atlas ti software. The results show, among other issues, the usefulness of the electronic portfolio for the development of new skills and abilities, in relation to the professional skills that students have to acquire.
\end{abstract}

Keywords: web 2.0; social software; internet; higher education; electronic portfolios 


\section{INTRODUCCIÓN}

En el nuevo contexto europeo de Educación Superior, en el que la calidad de los sistemas universitarios constituye una de las preocupaciones más destacadas de los países comprometidos con políticas sociales avanzadas (Imbernón, Silva y Guzmán, 2011), es necesario un cambio metodológico y evaluador orientado (Aguaded, López Meneses y Jaén, 2013) que potencie tanto la formación integral de los estudiantes, a través del desarrollo de sus competencias sociales, intelectuales y tecnológicas, como la reflexión colectiva mediante procesos de evaluación formativa (López Meneses, Domínguez, Álvarez y Jaén, 2011). Las Universidades deben cambiar y la gran mayoría así lo están haciendo, centrando los procesos de formación en las características y necesidades de los estudiantes, incorporando escenarios flexibles y abiertos para la formación y el aprendizaje y elaborando procesos de heteroevaluación entre los estudiantes para potenciar un desarrollo profesional más óptimo en perspectiva internacional (Farmer, Yue y Brooks, 2008).

Por otra parte, es indudable que la incorporación de las Tecnologías de la Información y la Comunicación (TIC) ha suscitado un proceso de cambio sin precedentes que ha afectado a las actividades y saberes de la humanidad y particularmente al medio universitario (Lagunes-Domínguez et al., 2015). Las nuevas tendencias tecnológicas emergentes están influyendo y transformando los procesos formativos, desde cambios organizacionales, favoreciendo la interacción entre los miembros de la comunidad universitaria y especialmente, transformando las estrategias didácticas para la formación competencial, empleabilidad y construcción del conocimiento globalizado, posibilitando el trabajo colaborativo de los estudiantes (EACEA/Eurydice, 2011; Centeno y Cubo, 2013).

Asimismo, el progresivo aumento de aplicaciones basadas en la web también se está consolidando como un medio relevante que puede implementar nuevas funciones en los procesos formativos (Pulichino, 2006; Saeed et al., 2009). Shang et al. (2011) señalan que la incorporación de la web 2.0 a la educación implica que los procesos formativos tengan un carácter más social, dinámico, interactivo, creativo y personal.

Cada vez se hace más énfasis en el desarrollo de propuestas didácticas que involucren trabajo colaborativo, toda vez que éste resulta relevante para el aprendizaje significativo (Bravo et al, 2016). Actualmente se priorizan tanto el establecimiento de un sistema de enseñanza que favorezca la formación integral de los estudiantes orientado a cubrir las demandas socio-laborales y la formación a lo largo de la vida (Arís y Comas, 2011), como la reformulación de metodologías aplicadas en las aulas centrando su énfasis en el proceso de aprendizaje y dotando de mayor protagonismo a los estudiantes (Barberà et al., 2009; Salmerón et al., 2010).

En este sentido los portafolios pueden y deben jugar un papel importante en los procesos de innovación metodológica, evaluación e investigación educativa (Aguaded et al., 2010; Aguaded et al., 2013) y, de hecho, están generando en los últimos años un enorme interés e inversión económica en las universidades por su gran potencial para el estudiantado y el profesorado (Shroff et al., 2011; Trent y Shroff, 2012; Coffey y Ashford-Rowe, 2014). Diferentes estudios (Johnson y DiBiase, 2004; Mansvelder et al., 2007) demuestran que es muy interesante la integración de los portafolios en la cultura universitaria para la innovación educativa y la reflexión en contextos de formación de futuros profesionales de la Educación.

En la literatura científica se identifican varios beneficios adicionales de los portafolios en el desarrollo profesional, tales como contribuir al desarrollo de habilidades para la reflexión y autoevaluación, ayudar a la selección de la información, facilitar las prácticas de colaboración o el cultivo de los conocimientos y creencias sobre la profesión y la práctica docente (Darling-Hammond y Snyder, 2000). Además, éstos permiten la posibilidad de compartir ideas en una comunidad de aprendizaje (Jafari y Kaufman, 2006; Cobos y Vallejos, 2011), el desarrollo del pensamiento reflexivo (Klenowski, 2005) y pueden favorecer el desarrollo profesional (Barrett, 2001).

Son una de las mejores herramientas utilizadas para desarrollar las competencias en estudiantes (Poyatos y Allan, 2004) y pueden favorecer la reflexión, la autoevaluación, la metacognición, la organización y la autorregulación del conocimiento que se construye sobre el mundo y sobre uno mismo (Alcaraz, 2016). Además, como indican Parkes y Kajder (2010) y Parkes et al., (2013), los portafolios facilitan la reflexión introspectiva sobre la propia docencia impartida con el estudiantado y favorecen un desarrollo profesional permanente que permite establecer relaciones con las experiencias pasadas y con el aprendizaje futuro.

Para Pérez-Gómez (2016) posibilitan el aprendizaje autónomo, el estudio independiente, las capacidades de autorregulación y la capacidad de aprender cómo aprender. Además permiten demostrar el crecimiento de la consecución de los resultados del aprendizaje para medir lo que los estudiantes han aprendido y son capaces de hacer cuando completen su titulación (Tubaishat, 2015). 
En este estudio se ha empleado el portafolio electrónico como un sistema digital que permite a los usuarios documentar competencias, eventos y planes que son relevantes para ellos en un contexto escolar, universitario y formativo (Barberà, 2008; Medina Rivilla, Domínguez Garrido y Sánchez Romero, 2013). El portafolio es accesible, fácil de consultar, descargar, permite una revisión transversal y evolutiva del trabajo del estudiante (Johnson et al., 2006; González Sánchez y García Muiña, 2011). En este sentido, en la literatura especializada sobre los portafolios en formato electrónico, se ha demostrado que más allá de la recopilación de evidencias de desempeño en la forma de artefactos digitalizados, lo más significativo es la naturaleza reflexiva de la herramienta (Chen y Light, 2010; Joyes et al., 2010).

De hecho, Alexiou y Paraskeva (2010) y Cheng y Chau (2013) afirman que el proceso de enseñanzaaprendizaje utilizando e-portafolios evidencia que el soporte digital de la herramienta y su relación con el aprendizaje autorregulado puede proporcionar un mejoramiento del proceso reflexivo. Por otra parte, bajo una perspectiva didáctica socio-constructivista e investigadora, consideramos que estos son una interesante alternativa metodológica para la innovación didáctica y la evaluación pedagógica al motivar el razonamiento reflexivo de la acción educativa, el desarrollo de competencias metacognitivas y, en última instancia, el desarrollo profesional.

En concreto, se utilizó un portafolio electrónico de carácter individual diseñado a través de un Edublog mediante el servicio Blogger, que se encuentra en el puesto decimoctavo del listado de herramientas 2.0 para el aprendizaje, recopilado por el Centro Learning \& Performance Technologies (C4LPT) (Hart, 2015). Hemos seleccionado esta herramienta porque la consideramos, como Cobos y Vallejos (2011), una buena puerta de entrada para los estudiantes a la llamada web social que les puede ayudar a mejorar sus destrezas informáticas y comunicativas, así como contribuir positivamente a desarrollar las asignaturas desde una perspectiva constructivista.

Como muchos autores han señalado, los blogs son publicaciones en red que permiten a los usuarios crear y editar el contenido de una página web con un mínimo de conocimientos técnicos (Huffaker, 2005). Son fáciles de utilizar en el contexto universitario, favorecen habilidades de aprendizaje de orden superior, el desarrollo de un papel activo del estudiante y fomentan la construcción de comunidades de conocimientos (Cabero, López-Meneses y Ballesteros, 2009; Aguaded, López-Meneses y Alonso, 2010). Para Roig son, de hecho, el recurso tecnológico idóneo para la elaboración de portafolios (Roig, 2009).

Merece la pena recordar la argumentación de Cambridge $(2009,2010)$ con relación a la construcción de dos personalidades en los e-portafolios para representar la imagen auténtica de su autor: el yo en red (networked self) y el yo sinfónico (symphonic self). El enfoque del yo en red es aquel por el que se conectan tareas y dispositivos que permitan obtener evidencias de aprendizaje y, a su vez, una autorreflexión del aprendizaje por parte del usuario. La selección de las herramientas susceptibles de emplear por el individuo es en sí un proceso que implica un autoconocimiento del propio estudiante. Todas estas actividades se integran en la rutina diaria como un diario cronológico que evidencia lo aprendido.

Por su parte, el yo sinfónico reorganiza toda esta actividad dispar en bloques temáticos de forma que el estudiante puede proporcionar autenticidad e integridad a su identidad, lo que representa en sí un objetivo y un reto para los creadores de e-portafolios. Este tipo de actividad requiere de tiempo y suficiente calma para fomentar una reflexión profunda que pueda ayudar a interconectar dispositivos y evidenciar y proporcionar una visión global de todo el proceso de aprendizaje. Por lo tanto, los "yos" de Cambridge precisan de una selección de herramientas que puedan mejorar el e-portafolio en sí y se considera que los blogs y las herramientas de software social pueden mejorar la construcción del yo en red.

Con este trabajo pretendemos dar continuidad y profundizar en la investigación "Innovación docente 2.0 con Tecnologías de la Información y la Comunicación (TIC) en el Espacio Europeo de Educación Superior”, situada en el marco de la Acción 2 de Proyectos de Innovación y Desarrollo Docente, auspiciados por el Vicerrectorado de Docencia y Convergencia Europea de la Universidad Pablo de Olavide, de Sevilla (España). En ella se describen diferentes prácticas universitarias orientadas al desarrollo competencial individual y por grupos interuniversitarios mediante el diseño, producción e implementación de e-actividades relacionadas con el software social.

Los principales objetivos de la experiencia universitaria innovadora eran los siguientes: reflexionar sobre las principales teorías contemporáneas de la Educación y su relación con la práctica profesional actual de la Educación Social, realizar búsquedas de información y recursos en Internet, conocer y utilizar software social 2.0. (cuadernos de bitácora), describir los aspectos sociales más notables en la presentación del blog resultante de la práctica de innovación, tutorizar y ayudar a los estudiantes en sus tomas de decisiones, potenciar la capacidad crítica y autocrítica del estudiante y realizar procesos de autoevaluación de la práctica educativa. 
Aunque el uso de los portafolios electrónicos puede llegar a ser una forma novedosa para introducir cambios significativos en el aprendizaje de los estudiantes, éste tipo de práctica está cada vez más difundida y, como tal, no presenta una novedad global. Sin embargo, consideramos que la coordinación de las dos asignaturas implicadas (una del área de Teoría e Historia de la Educación y otra más tecnológica, del área de Didáctica y Organización Educativa), así como la articulación de las actividades en la construcción de nuevas competencias profesionales en los estudiantes, presentan un valor agregado a este trabajo.

La investigación presentada se enfoca a la comprensión de una práctica educativa innovadora basada en la conceptualización de las principales ideas, reflexiones y repercusiones didácticas de los autores más relevantes del corpus de la Historia de la Educación Contemporánea. Más concretamente, el objetivo general de la investigación se concreta en el análisis de los portafolios electrónicos de los estudiantes en base a tres dimensiones: aspectos técnicos, aspectos educativos y aspectos sociales.

\section{EL CASO DE ESTUDIO}

El escenario del estudio se desarrolla durante los meses de marzo y abril del curso académico 2013-14 y 2014-15 en dos asignaturas: Tecnologías de la Información y la Comunicación en Educación Social y Fundamentos Pedagógicos de la Educación Social, correspondientes ambas al primer año de la titulación del Grado de Educación Social (2013-14) y Doble Grado de Educación Social y Trabajo Social (2014-15) de la Facultad de Ciencias Sociales de la Universidad Pablo de Olavide, de Sevilla (España).

Referente al programa de estudios, la asignatura de Tecnologías de la Información y la Comunicación en Educación Social, pertenece al área de Didáctica y Organización Educativa y se articula alrededor de cuatro núcleos de contenidos: Didáctica, Currículo y TIC en Educación Social, Recursos didácticos-tecnológicos y su utilización educativa, Evaluación y Temas sociales/transversales. En concreto, este trabajo hace alusión al segundo bloque temático que tiene como objeto de estudio los recursos didácticos-tecnológicos y su utilización educativa. El organigrama conceptual de los bloques de contenidos de la asignatura se puede consultar en este enlace: www.mindomo.com/view.htm?m=6cd2bf5f280e4e7bb7cca11b77b8beb1

En cuanto al programa de la asignatura Fundamentos Pedagógicos de la Educación Social, que pertenece al área de Teoría e Historia de la Educación, ésta se estructura en cuatro bloques de contenido: Pedagogía General: fundamentos epistemológicos de la Educación, Fundamentos históricos de la Educación, Teorías contemporáneas de la Educación y Agentes e instituciones educativas contemporáneas. El bloque trabajado en esta experiencia es el referido a las Teorías contemporáneas de la Educación. La articulación pormenorizada de estos bloques de contenido con las unidades temáticas concretas a trabajar, se puede consultar en este enlace: www.mindomo.com/es/mindmap/mapa-de-contenidos-de-la-asignatura 63e2cab6308341e7a1858df334982bbe

Ambas asignaturas se imparten, como ya se ha dicho, en el primer curso de Grado y forman parte del módulo denominado "Bases conceptuales y contextuales de la Educación Social". La asignatura de Fundamentos Pedagógicos contribuye a la adquisición y comprensión de los fenómenos y procesos sociales que requieren intervención socioeducativa desde una perspectiva pedagógica, proporcionando las bases teóricas e históricas que permiten acercarse a una comprensión amplia de los fenómenos socio-educativos.

En este sentido, se reflexiona sobre los desarrollos científicos, tecnológicos, sociales y socio-pedagógicos que han experimentado las distintas teorías de la educación a lo largo de la Historia, se dan a conocer los distintos agentes educativos, se valora su importancia en la formación integral de los ciudadanos y se construyen propuestas educativas desde una perspectiva de igualdad, equidad y compromiso ético.

En la asignatura de TIC se analiza críticamente la Sociedad de la Información y el Conocimiento, se dan a conocer diferentes soportes de la información y de sus diversos lenguajes, se expresan discursos audiovisuales críticos a través de aplicaciones del software social y se desarrollan nuevas alternativas para la expansión del conocimiento global.

Para ello se trata de facilitar el aprendizaje a lo largo de la vida, a través de la construcción de comunidades de conocimiento colectivos de información en la Sociedad de la Comunicación, con el fin de favorecer el desarrollo personal y profesional de los estudiantes, se dan a conocer las potencialidades que ofrecen las TIC en el contexto de la acción socioeducativa y sus procesos de formación, gestión y organización y se dan las claves para diseñar procesos de creación de redes sociales de conocimiento e innovación para la intervención socioeducativa. 
El diseño pedagógico de ambas asignaturas se ha desarrollado al albur de los cambios sugeridos por el Espacio Europeo de Educación Superior que, a pesar de las muchas contradicciones que hayan podido comportar, han abierto la oportunidad de plantear cambios metodológicos que contribuyen a hacer una formación capacitadora para trabajar en escenarios de complejidad (Rosa, Riberas, Navarro-Segura y Vilar, 2015). El desafío para las universidades, en este marco, reside en rediseñar sus matrices formativas alrededor de las competencias de salida más que alrededor de las tradicionales asignaturas (Icarte y Lávate, 2016).

La articulación de las competencias desarrolladas en ambas asignaturas se puede ver en la tabla 1 . Para el desarrollo de las mismas, ponemos el foco en la lógica constructivista del aprendizaje, que incide en el protagonismo del estudiante, en la lógica de construir a partir de lo ya aprendido, de hacerlo en un contexto social con un elevado grado de autoconciencia por parte del mismo (Rosa, Navarro-Segura y López, 2014).

Tabla 1. Articulación de las competencias desarrolladas en ambas asignaturas

\begin{tabular}{|c|c|c|c|}
\hline \multirow{9}{*}{$\begin{array}{l}\text { Competencias } \\
\text { Generales }\end{array}$} & Competencias a desarrollar en las asignaturas & $\begin{array}{l}\text { Fundamentos } \\
\text { Ped. de la Ed. } \\
\quad \text { Social }\end{array}$ & $\begin{array}{l}\text { TIC en } \\
\text { Educación } \\
\text { Social } \\
\end{array}$ \\
\hline & $\begin{array}{l}\text { Desarrollar competencias en el campo de las nuevas tecnologías y la } \\
\text { gestión de la innovación }\end{array}$ & & $\mathrm{x}$ \\
\hline & $\begin{array}{l}\text { Desarrollar habilidades de resolución de problemas y toma de } \\
\text { decisiones }\end{array}$ & & $\mathrm{x}$ \\
\hline & $\begin{array}{l}\text { Desarrollar habilidades de creatividad, ampliando las posibilidades } \\
\text { convencionales tanto de comprensión y de juicio como de aplicación } \\
\text { en la resolución de problemas y toma de decisiones }\end{array}$ & $\mathrm{x}$ & \\
\hline & $\begin{array}{l}\text { Capacidad de reunir e interpretar datos relevantes para emitir juicios } \\
\text { sobre temas relevantes de índole social, científica o ética }\end{array}$ & $\mathrm{x}$ & \\
\hline & $\begin{array}{l}\text { Desarrollar las competencias implicadas para el dominio correcto de } \\
\text { la lengua española }\end{array}$ & $\mathrm{x}$ & \\
\hline & Desarrollar apertura hacia al aprendizaje a lo largo de la vida & $\mathrm{x}$ & $\mathrm{x}$ \\
\hline & Capacidad para trabajar en equipo & $\mathrm{x}$ & $\mathrm{x}$ \\
\hline & $\begin{array}{l}\text { Afrontar los deberes y dilemas éticos con espíritu crítico ante las } \\
\text { nuevas demandas y formas de exclusión social que plantea la } \\
\text { sociedad del conocimiento a la profesión del educador/a social }\end{array}$ & $\mathrm{x}$ & $x$ \\
\hline \multirow{5}{*}{$\begin{array}{l}\text { Competencias } \\
\text { Específicas }\end{array}$} & $\begin{array}{l}\text { Conocer, comprender y desarrollar las posibilidades que ofrecen las } \\
\text { TIC en el ámbito de la intervención socioeducativa y sus procesos de } \\
\text { gestión y organización }\end{array}$ & $\mathrm{x}$ & $x$ \\
\hline & $\begin{array}{l}\text { Adquirir las habilidades, destrezas y actitudes para la intervención } \\
\text { socioeducativa }\end{array}$ & $\mathrm{x}$ & $\mathrm{x}$ \\
\hline & $\begin{array}{l}\text { Conocer y comprender de forma crítica las bases teóricas y } \\
\text { metodológicas que desde perspectivas pedagógicas, sociológicas, } \\
\text { psicológicas sustentan los procesos socioeducativos, así como los } \\
\text { marcos legislativos que posibilitan, orientan y legitiman la acción del } \\
\text { Educador y Educadora social }\end{array}$ & $\mathrm{x}$ & \\
\hline & $\begin{array}{l}\text { Analizar, conocer y comprender la trayectoria de la Educación Social } \\
\text { y la configuración de su campo e identidad profesional que cultural e } \\
\text { históricamente ha ido adquiriendo la profesión del Educador y } \\
\text { Educadora Social, así como las características de las instituciones y } \\
\text { organizaciones en las que desempeña su trabajo, con objeto de } \\
\text { configurar su campo e identidad profesional }\end{array}$ & $\mathrm{x}$ & \\
\hline & $\begin{array}{l}\text { Identificar los fundamentos de la gestión de procesos de calidad e } \\
\text { innovación en instituciones vinculadas con la intervención } \\
\text { socioeducativa }\end{array}$ & $\mathrm{x}$ & \\
\hline
\end{tabular}

Los estudiantes tenían que desarrollar un trabajo en equipo sobre uno de los pedagogos contemporáneos incluidos en el programa de la asignatura Fundamentos Pedagógicos de la Educación Social. En concreto, se trataba de construir un documento para poner a disposición del resto de compañeros a través del Aula Virtual de la asignatura, con una síntesis que recogiera los siguientes aspectos básicos: contextualización socio-histórica del autor, principales aportes pedagógicos y actualidad e impacto de sus teorías para la práctica profesional de la Educación Social. Además, también en equipo, debían diseñar e implementar una explicación práctica de este trabajo para presentar al conjunto del grupo-clase y para ello, apoyarse en las 
posibilidades de las TIC que se trabajan en profundidad en la otra asignatura. Se insta a los estudiantes a ser creativos y utilizar todos los recursos que deseen de cara a la exposición ante sus compañeros.

Para contextualizar didácticamente el desarrollo de la experiencia universitaria, en la asignatura denominada "Tecnologías de la información y la comunicación en Educación Social", se impartieron dos sesiones de hora y media de duración durante el mes de marzo en el aula de informática para conocer las características más significativas de los edublogs. Posteriormente, cada educando enviaría su enlace al blog de la asignatura: http://blogosfera3000.blogspot.com.es/ e implementaría una pestaña nueva en su Edublog personal denominada "Fundamentos Pedagógicos" con los contenidos en formato multimedia que hubiera utilizado en las exposiciones de los trabajos defendidos en la otra asignatura. A modo de ejemplo, se puede consultar el edublog realizado por una estudiante del Grado de Educación Social en: http://carolinatic3000.blogspot.com.es/p/blog-page.html

\section{METODOLOGÍA}

La metodología de investigación que empleamos fue flexible, interactiva, holística, subjetiva y orientada a la mejora del proceso educativo, en la línea que propugnan Stufflebeam y otros autores (Escudero, 2003). La muestra estuvo conformada por un total de 86 estudiantes correspondientes a las dos asignaturas: Tecnologías de la Información y la Comunicación y Fundamentos Pedagógicos de la Educación Social, correspondientes al primer curso de la titulación de Grado de Educación Social y del Doble Grado en Educación Social y Trabajo Social de la Universidad Pablo de Olavide. Para analizar los diferentes documentos elaborados por los estudiantes (comentarios realizados en los edublogs del estudiantado) a lo largo de la experiencia didáctica, tomamos como marco de referencia las pautas establecidas por Bogdan y Biklen (1992) y Miles y Huberman (1994).

En una primera fase procedimos a la reducción de datos mediante la categorización y codificación de la información obtenida. La categorización implicó simplificar y seleccionar la información para hacerla más manejable. Para ello seguimos los siguientes pasos: separación de unidades para identificar segmentos significativos de información sobre los respectivos ámbitos de conocimiento; identificación y clasificación de las unidades para agruparlas conceptualmente en grupos que compartían un mismo tópico con significado; síntesis y agrupamiento de las diferentes unidades de información. Durante la codificación identificamos cada unidad textual con su categoría correspondiente a través de un procedimiento mixto (inductivodeductivo) para proceder seguidamente a su recuento frecuencial.

El proceso de análisis se completó con una segunda fase en la que interpretamos las diferentes unidades de información categorizadas, ordenando de modo sistemático la información obtenida para facilitar la fase de inferencia y explicación de los resultados que detallamos a continuación. Los datos utilizados para este estudio se centran en los portafolios electrónicos realizados por el alumnado y recopilados en el edublog de la asignatura. El análisis de los datos se realizó con ayuda del programa informático ATLAS.ti 6.2. Este software facilita el desarrollo de la fase instrumental y de codificación, ya que se orienta a la interpretación de textos e imágenes. Además, favorece la gestión de anotaciones, conceptos y estructuras complejas evidenciando relaciones emergentes entre los mismos (Muhr, 1991; Barry, 1998; Friese, 2014).

En cuanto al proceso de reducción y estructuración teórica de la información se tuvo como marco de referencia las fases de identificación de unidades de información: codificación, categorización, interpretación e inferencia (Miles y Huberman, 1994). En relación al proceso de codificación, cabe mencionar que el sistema de categorías fue elaborado ad hoc, en base a los códigos que subyacían en el análisis de los portafolios electrónicos de los alumnos. Es decir, se siguió un proceso de codificación inductivo dando lugar a tres grandes dimensiones de análisis: aspectos técnicos, educativos y sociales. En la tabla 2 se ha indicado el sistema de categorías en su conjunto con la correspondiente descripción de las mismas.

Posteriormente, entramos a analizar la evaluación de los trabajos, mediante la técnica de análisis de contenido, a través de los textos escritos por los propios estudiantes en sus blogs. Así, mediante un análisis interno e intensivo de los documentos, procuramos destacar su sentido y caracteres fundamentales. Esta crítica interna se basa en una interpretación personal y subjetiva, cobrando especial relevancia en lo que se refiere a la medición de la motivación, como apunta López-Noguero (2002).

Finalmente cabe aclarar, entre las limitaciones del estudio, que entendemos la evaluación de competencias profesionales como un proceso realmente complejo que requiere de una mayor profundización evaluativa tanto temporal como metodológicamente. En este sentido, este trabajo ha de entenderse como una aproximación tentativa, susceptible de ser ampliada y complementada en ulteriores trabajos que pretendemos seguir desarrollando en la misma línea. 
Tabla 2. Descripción del sistema de categorías utilizado

\begin{tabular}{|c|c|c|}
\hline Dimensión & Categoría & Descripción \\
\hline \multirow{4}{*}{$\begin{array}{l}\text { Aspectos } \\
\text { técnicos }\end{array}$} & Color & Categoría donde se evidencia el uso de colores en el blog del alumno. \\
\hline & Tipo de fuente & $\begin{array}{l}\text { Categoría con información relativa al tipo de fuente utilizada por el } \\
\text { alumno. }\end{array}$ \\
\hline & Recurso textual & $\begin{array}{l}\text { Categoría dónde se refleja los recursos utilizados por el alumno para } \\
\text { exponer la información. }\end{array}$ \\
\hline & Recurso multimedia & $\begin{array}{l}\text { Categoría que hace referencia al tipo de recurso multimedia utilizado en } \\
\text { el blog del alumno. }\end{array}$ \\
\hline \multirow[t]{8}{*}{$\begin{array}{l}\text { Aspectos } \\
\text { educativos }\end{array}$} & Tema principal & $\begin{array}{l}\text { Categoría que recoge al autor que ha escogido el alumno para realizar } \\
\text { su trabajo. }\end{array}$ \\
\hline & $\begin{array}{l}\text { Presentación de la } \\
\text { información }\end{array}$ & $\begin{array}{l}\text { Categoría relacionado con la variedad y diversidad de recursos } \\
\text { utilizados por el alumno en la presentación de la información. }\end{array}$ \\
\hline & Evaluación & $\begin{array}{l}\text { Categoría dónde se refleja la evaluación que realiza el alumno de los } \\
\text { contenidos que presenta o de la experiencia que ha supuesto la } \\
\text { actividad. }\end{array}$ \\
\hline & Organización & $\begin{array}{l}\text { Categoría que hace referencia a la ordenación de la información en el } \\
\text { blog del alumno. }\end{array}$ \\
\hline & Claridad y síntesis & $\begin{array}{l}\text { Categoría con información relativa a la claridad y síntesis en la } \\
\text { presentación de la información por parte del alumno. }\end{array}$ \\
\hline & Ortografía & $\begin{array}{l}\text { Categoría dónde se evidencia la ortografía del alumno en la redacción y } \\
\text { presentación de la información. }\end{array}$ \\
\hline & Estructura & $\begin{array}{l}\text { Categoría en la que se plasma la relevancia que otorga el alumno a } \\
\text { cada temática de la estructura. }\end{array}$ \\
\hline & Profundidad & $\begin{array}{l}\text { Categoría en la que se plasma la profundidad del trabajo realizado por } \\
\text { el alumno. }\end{array}$ \\
\hline \multirow[t]{5}{*}{$\begin{array}{l}\text { Aspectos } \\
\text { sociales }\end{array}$} & Enlaces a otras webs & $\begin{array}{l}\text { Categoría con información relativa al número de enlaces a otras webs } \\
\text { que posee el blog del alumno. }\end{array}$ \\
\hline & Seguidores del blog & $\begin{array}{l}\text { Categoría que hace referencia al número de seguidores del blog del } \\
\text { alumno. }\end{array}$ \\
\hline & Visitas al blog & $\begin{array}{l}\text { Categoría dónde se refleja el número de visitas que ha recibido el } \\
\text { alumno en su blog. }\end{array}$ \\
\hline & Comentarios en el blog & $\begin{array}{l}\text { Categoría que recoge la cantidad de comentarios recibidos en el blog } \\
\text { del alumno. }\end{array}$ \\
\hline & Información personal & $\begin{array}{l}\text { Categoría con información relativa a la información personal compartida } \\
\text { por el alumno en su blog. }\end{array}$ \\
\hline
\end{tabular}

\section{RESULTADOS Y DISCUSIÓN}

Con la finalidad de obtener una mayor comprensión de los resultados alcanzados en la investigación se exponen los mismos en base a cada una de las dimensiones analizadas. En primer lugar, se abarca la dimensión de "Aspectos técnicos" del análisis de los blogs educativos de los alumnos, que se expone en la tabla 3. Podemos destacar la utilización de recursos textuales y multimedia por la mayoría del alumnado, lo cual facilita la transmisión del conocimiento al ser presentado en diversos formatos. En este sentido, resulta interesante el uso de herramientas de apoyo que favorecen la visibilidad y accesibilidad al blog, en la línea de lo que apuntan estudios anteriores como el de Díaz y Rojas (1999).

A la luz de los resultados obtenidos, resulta destacable el apoyo de la gran mayoría del alumnado en el uso de herramientas textuales y multimedia en sus presentaciones virtuales, aunque un pequeño porcentaje opta por la exposición meramente textual. Asimismo, de todas las herramientas digitales disponibles en la web, los alumnos han optado por la utilización de las que se plasman en los resultados. En base al uso que el alumnado les otorgó en su blog podemos englobarlas en dos bloques diferenciados, las herramientas utilizadas en la presentación del contenido y las que realizan funciones de apoyo al blog. De este modo, quedarían descritas como se aprecia en la figura 1. En relación a las herramientas digitales utilizadas en la presentación de la información, destaca la variedad de recursos escogidos aun siendo bastante notable la elección de Prezi y YouTube. Por otro lado, los recursos de apoyo más recurrentes están relacionados con la visibilidad del blog (por ejemplo, inclusión del Código QR), evidenciando el desarrollo de la competencia sobre utilización de TIC. 
Tabla 3. Resultados numéricos de la dimensión "Aspectos técnicos"

\begin{tabular}{|c|c|c|c|c|}
\hline Categoría & Subcategoría & Cód. & Frec. & $\%$ \\
\hline \multirow[t]{3}{*}{ Color } & Colores suaves (tonos claros, pastel) & CSU & 56 & 65,2 \\
\hline & Colores fuertes (colores oscuros, opacos) & CFU & 18 & 20,9 \\
\hline & Colores de alto contraste (contrastan dos colores, dificultando la lectura) & CAC & 12 & 13,9 \\
\hline \multirow[t]{2}{*}{ Tipo de fuente } & Fuente clásica (Fuentes clásicas de uso en la web) & FCL & 80 & 93 \\
\hline & $\begin{array}{l}\text { Fuente llamativa (Fuentes que llaman la atención por no ser utilizadas } \\
\text { habitualmente) }\end{array}$ & FLL & 6 & 7 \\
\hline \multirow[t]{2}{*}{ Recurso textual } & Textual y multimedia (Si utiliza ambos tipos de recursos) & TYM & 82 & 95,4 \\
\hline & Sólo textual (Si sólo utiliza el recurso textual) & ST & 4 & 4,6 \\
\hline \multirow{12}{*}{$\begin{array}{l}\text { Recurso } \\
\text { multimedia }\end{array}$} & Código QR & QR & 60 & 69,8 \\
\hline & Videojuego & VJU & 53 & 61,6 \\
\hline & Prezi & $\mathrm{PZI}$ & 52 & 60,5 \\
\hline & YouTube & YT & 25 & 29,1 \\
\hline & Traductor & TDT & 13 & 15,1 \\
\hline & Taxgedo & TXG & 8 & 9,3 \\
\hline & Calendario & CLD & 8 & 9,3 \\
\hline & Imagen & IMG & 7 & 8,1 \\
\hline & Slideshare & SLS & 6 & 7 \\
\hline & Videocomic & VCO & 4 & 4,6 \\
\hline & Mindomo & MDM & 4 & 4,6 \\
\hline & Libro electrónico & LE & 4 & 4,6 \\
\hline
\end{tabular}

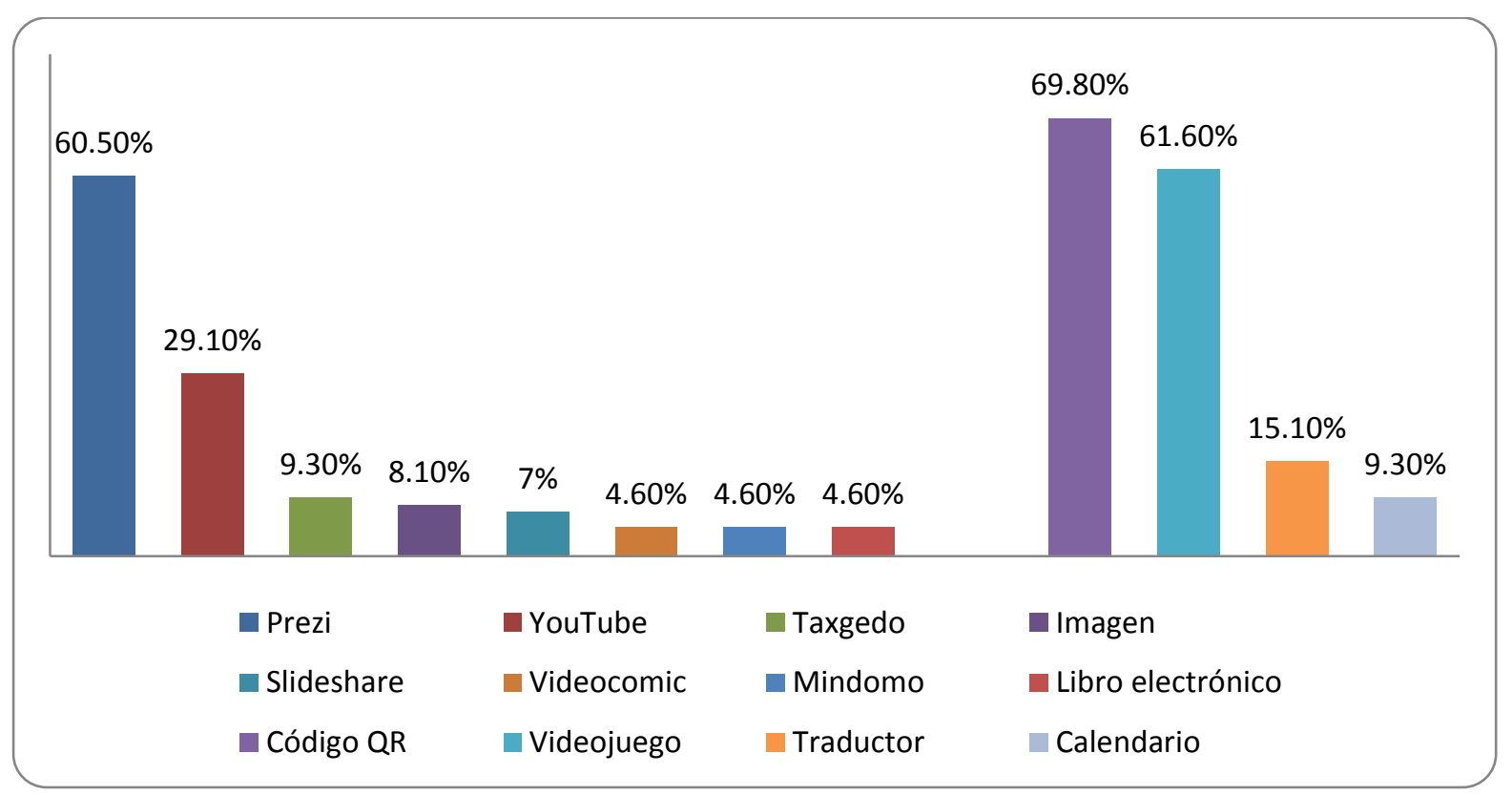

Fig. 1. Recursos multimedia utilizados en la presentación o de apoyo al blog

Respecto a la dimensión de "Aspectos educativos", cuyos resultados numéricos apreciamos en la tabla 4, es destacable la elección por parte de los estudiantes de la autora María Montessori, seguida de Anton Makarenko y John Dewey. En contraposición a ello, los menos escogidos fueron Alexander Neill y Francesc Ferrer i Guàrdia. Asimismo, se evidencia que la mayoría de estudiantes utilizan multitud de recursos en la exposición del autor escogido. El 7\% que utiliza un único recurso correlaciona directamente con el alumnado que plasma la información únicamente a través de texto. 
Tabla 4. Resultados numéricos de la dimensión "Aspectos educativos"

\begin{tabular}{|c|c|c|c|c|c|}
\hline Categoría & \multicolumn{2}{|l|}{ Subcategoría } & Cód. & Frec. & $\%$ \\
\hline \multirow[t]{8}{*}{ Tema principal } & \multicolumn{2}{|l|}{ Montessori } & MON & 19 & 22,1 \\
\hline & \multicolumn{2}{|l|}{ Makarenko } & MAK & 14 & 16,3 \\
\hline & \multicolumn{2}{|l|}{ Dewey } & DEW & 12 & 13,9 \\
\hline & \multicolumn{2}{|l|}{ Illich } & ILL & 10 & 11,6 \\
\hline & \multicolumn{2}{|l|}{ Freire } & FRR & 9 & 10,5 \\
\hline & \multicolumn{2}{|l|}{ Freinet } & FRN & 9 & 10,5 \\
\hline & \multicolumn{2}{|l|}{ Ferrer i Guàrdia } & FYG & 8 & 9,3 \\
\hline & \multicolumn{2}{|l|}{ Neill } & $\mathrm{NEI}$ & 5 & 5,8 \\
\hline \multirow{2}{*}{$\begin{array}{l}\text { Presentación de la } \\
\text { información }\end{array}$} & \multicolumn{2}{|c|}{ Diversidad de recursos (Utilizan más de un recurso en su exposición) } & DRC & 80 & 93 \\
\hline & \multicolumn{2}{|c|}{ Único recurso (Sólo utilizan un recursos en la exposición del tema) } & URC & 6 & 7 \\
\hline \multirow[t]{3}{*}{ Evaluación } & \multicolumn{2}{|c|}{ Ninguna evaluación (No se realiza ninguna evaluación) } & $\mathrm{NE}$ & 75 & 87,2 \\
\hline & \multicolumn{2}{|c|}{$\begin{array}{l}\text { Evaluación de la experiencia (Evalúan la experiencia que ha supuesto } \\
\text { realizar el trabajo) }\end{array}$} & EEX & 10 & 11,6 \\
\hline & \multicolumn{2}{|c|}{$\begin{array}{l}\text { Evaluación de los conocimientos (Evalúan la relevancia de los } \\
\text { conocimientos adquiridos) }\end{array}$} & ECO & 1 & 1,2 \\
\hline \multirow[t]{2}{*}{ Organización } & \multicolumn{2}{|c|}{$\begin{array}{l}\text { Ordenación adecuada (La información está bien ordenada, facilitando } \\
\text { su lectura) }\end{array}$} & ODA & 86 & 100 \\
\hline & \multicolumn{2}{|c|}{$\begin{array}{l}\text { Uso de etiquetas (Utilizan etiquetas para facilitar la navegación y la } \\
\text { búsqueda de información) }\end{array}$} & UEQ & 0 & 0 \\
\hline \multirow[t]{2}{*}{ Claridad y síntesis } & \multicolumn{2}{|c|}{ Claridad (La exposición de la información es clara) } & CLA & 86 & 100 \\
\hline & \multicolumn{2}{|c|}{ Síntesis (Resumen la información de manera adecuada) } & SIN & 86 & 100 \\
\hline \multirow[t]{2}{*}{ Ortografía } & \multicolumn{2}{|c|}{ Correcta ortografía (La ortografía es correcta) } & COR & 86 & 100 \\
\hline & \multicolumn{2}{|c|}{ Faltas de ortografía (Cometen faltas de ortografía) } & $\mathrm{FAO}$ & 0 & 0 \\
\hline \multirow[t]{6}{*}{ Estructura } & \multirow[t]{2}{*}{ Contextualización histórica } & $\begin{array}{l}\text { Correcta contextualización histórica } \\
\text { del autor }\end{array}$ & $\mathrm{CCH}$ & 85 & 98,8 \\
\hline & & $\begin{array}{l}\text { Escasa contextualización histórica del } \\
\text { autor }\end{array}$ & $\mathrm{ECH}$ & 1 & 1,2 \\
\hline & \multirow[t]{2}{*}{$\begin{array}{l}\text { Principales teorías } \\
\text { pedagógicas }\end{array}$} & $\begin{array}{l}\text { Correcta en principales teorías } \\
\text { pedagógicas }\end{array}$ & CPT & 84 & 97,7 \\
\hline & & $\begin{array}{l}\text { Escasa en principales teorías } \\
\text { pedagógicas }\end{array}$ & EPT & 2 & 2,3 \\
\hline & \multirow[t]{2}{*}{ Aportaciones a la educación } & $\begin{array}{l}\text { Correcta en aportaciones a la } \\
\text { educación }\end{array}$ & CAE & 8 & 9,3 \\
\hline & & $\begin{array}{l}\text { Escasa en aportaciones a la } \\
\text { educación }\end{array}$ & EAE & 78 & 90,7 \\
\hline \multirow[t]{2}{*}{ Profundidad } & \multicolumn{2}{|c|}{$\begin{array}{l}\text { Repetición de hechos o ideas (La información expuesta es una mera } \\
\text { repetición de hechos o ideas) }\end{array}$} & $\mathrm{RHI}$ & 64 & 74,4 \\
\hline & $\begin{array}{l}\text { Visión crítica (Aportan su visi } \\
\text { autores) }\end{array}$ & sobre los hechos o ideas de los & VC & 22 & 25,6 \\
\hline
\end{tabular}

En cuanto a la evaluación cualitativa del trabajo, desarrollada a partir del análisis de contenido de los comentarios de los propios estudiantes, hay que de decir que ellos mismos destacan la importancia de conocer la vida y obra de los autores como base teórica posteriormente aplicable a su quehacer socioeducativo (conocer y comprender de forma crítica las bases teóricas y metodológicas desde perspectivas pedagógicas...): "gracias a este trabajo he aprendido que estudiar y conocer la vida de los autores no siempre es aburrido ya que hay autores que con su labor consiguen cosas que son muy importantes para la vida de hoy en día."; la valoración del trabajo en grupo (competencia: capacidad para trabajar en equipo): "Este que trata sobre Ferrer $i$ Guardia ha sido realizado por mis compañeras y yo, en las realización del 
mismo trabajamos todas por igual y colaborando en todo lo posible."; así como el fomento de la creatividad que ha supuesto: "Por último, quiero destacar que con este trabajo no sólo he aprendido sobre Ferrer $y$ Guàrdia, sino también a trabajar de forma creativa e innovadora a través de moviemaker y al ver otras formas de exposición realizadas por las demás compañeras".

En todos los casos, la ordenación del contenido mantiene coherencia en su desarrollo a lo largo del blog, poniendo de manifiesto y corroborando la investigación de Amestica et al (2016) en la que se muestra que se trata de una metodología de enseñanza-aprendizaje que permite compilar los trabajos realizados por los estudiantes en una asignatura, evidenciando su proceso de avance autorregulado y de evaluación en función de competencias y contenidos. Además, en sintonía con el resultado de la experiencia universitaria en la Universitat Politècnica de València, comprobamos también que la elaboración del portafolio electrónico ayuda al estudiantado a administrarse y gestionar la tarea académica, estableciendo pruebas significativas de su aprendizaje (Estelles, Barbera y Dema, 2013).

Por otro lado, cabe destacar que no se hallaron incorrecciones ortográficas en ninguna de las exposiciones de los alumnos, temática relacionada en ocasiones en la literatura científica con el uso de las TIC, y coherente con una de las competencias que se trata de desarrollar, como ya se ha mencionado anteriormente: dominio correcto de la lengua española.

Respecto a la contextualización histórica de los autores, la mayoría de los alumnos le dedican un importante espacio en sus presentaciones, exponiendo los aspectos más destacados en la vida de los mismos, ya sea en relación a su formación laboral y profesional: "María Montessori nació en Chiaravalle, Italia, el 3 de Marzo de 1870. Se traslada a Roma con 16 años junto a su familia para cursar estudios superiores. Se licencia en Medicina en 1896. Entra como ayudante a una clínica psiquiátrica dedicada a niños con discapacidad..."o en temáticas relacionadas con su vida familiar y personal "Era el segundo hijo de la familia. Aunque era muy enfermizo y débil, era un niño muy inteligente y se desarrollaba con normalidad. A los cinco años, ya sabía leer y tenía curiosidad por conocer la causa de todo lo que se encontraba a su alrededor. Su padre era un obrero pintor un tanto reservado y poco comunicativo. A diferencia de él, su madre era una mujer optimista y alegre, con gran sentido del humor. Conseguía mantener en la familia una atmósfera optimista."

Al igual que ocurre en la contextualización histórica, al apartado dedicado a las principales teorías pedagógicas de los autores, los estudiantes le otorgan un espacio significativo en sus presentaciones (competencias relacionadas con el análisis y comprensión de la trayectoria de la Educación Social y la configuración de su campo e identidad profesional cultural e histórica).

En este bloque, los alumnos exponen de manera general la visión de la educación que posee el autor en cuestión "Illich utiliza una perspectiva antropológica y fenomenológica a la hora de criticar la escuela. En su opinión, la escuela no educa, sino que acomoda a los alumnos a la burocracia del sistema. Así, el único saber valorado es el codificado en la escuela o en la educación oficial; así como, de manera específica, las propuestas educativas principales en las que basan sus actuaciones "Propone una reconstrucción de las prácticas morales y de las creencias, mediante la aplicación de métodos científicos y su conocimiento crítico: rechazo de la perspectiva de considerar a los niños como candidatos a adultos; educar no se trata de hacer que expertos en métodos educativos que enseñen a alumnos pasivos y receptivos, sino que debe existir iniciativa intelectual y capacidad de decisión."

Al enfocar su presentación en las aportaciones que realiza el autor a la educación, en general, o a la educación social, en particular, la mayoría de los estudiantes realizan exposiciones breves, presentando afirmaciones de carácter general acerca de su influencia en la actualidad: "Freire, no solo por la época que vivió, sino por su teoría, es un autor moderno, actual, reciente. Su teoría, o parte de ella, forma parte de la educación de países punteros en el desarrollos educativo y personal." o "Dewey mostró un sentido práctico para planificar y desarrollar un currículum integrado de las ocupaciones (actividades funciones ligadas al medio del niño), incluyendo previsiones de desarrollo del programa en ciclos temporales corto".

Finalmente, al observar la profundidad de las aportaciones de los estudiantes, se aprecia que, en torno a un $25 \%$ del alumnado, aporta una visión crítica a la presentación, relacionándola con aspectos cotidianos y actuales de nuestra vida: "Creo que es importante compartir lo que hacemos para que otras personas lo vean y comenten sobre lo que hemos trabajado, tal y como decía Freinet. Él es pionero en esto de las aplicaciones 2.0, defendía que todo debía ser compartido y enseñado y que no hace falta libros de texto para aprender." o bien, realizando un análisis crítico de las teorías y propuestas del autor: "La importancia de la escuela como uno de los escasos ámbitos públicos donde niños y jóvenes se encuentran para compartir aprendizajes. Necesitamos instituciones o ámbitos públicos dónde los niños se encuentren para compartir aprendizajes permitiendo la experimentación ¿Puede ser satisfecho esto por la escuela? Nos encontramos de frente a un problema antropológico que tiene que ver con el deseo de aprender." 
Asimismo, en relación a la visión crítica del alumnado en sus exposiciones (emitir juicios críticos), es destacable que ésta queda evidenciada en mayor medida en el epígrafe de las aportaciones del autor a la educación y a la educación social, ya que lo relacionan con la vigencia que poseen actualmente sus teorías, como observamos en las referencias expuestas anteriormente. Por último, respecto a la dimensión de "Aspectos sociales" del blog elaborado por los alumnos, se exponen los resultados numéricos en la tabla 5.

Tabla 5. Resultados numéricos de la dimensión "Aspectos sociales"

\begin{tabular}{|c|c|c|c|c|}
\hline Categoría & Subcategoría & Frec. & Cód. & $\%$ \\
\hline \multirow{3}{*}{$\begin{array}{l}\text { Enlaces a otras } \\
\text { webs }\end{array}$} & Más de 5 enlaces & 1 & MCE & 1,2 \\
\hline & Entre 1 y 5 enlaces & 6 & EUCE & 7 \\
\hline & Ningún enlace & 79 & $\mathrm{NE}$ & 91,9 \\
\hline \multirow[t]{3}{*}{ Seguidores del blog } & Más de 10 & 0 & MDS & 0 \\
\hline & Menos de 10 & 1 & MNS & 1,2 \\
\hline & Ningún seguidor & 0 & NS & 0 \\
\hline \multirow[t]{3}{*}{ Visitas al blog } & Más de 50 & 6 & MCV & 7 \\
\hline & Entre 10 y 50 & 0 & EDCV & 0 \\
\hline & Menos de 10 & 0 & MDV & 0 \\
\hline \multirow{2}{*}{$\begin{array}{l}\text { Comentarios en el } \\
\text { blog }\end{array}$} & Algún comentario & 0 & $\mathrm{ACM}$ & 0 \\
\hline & Ningún comentario & 86 & $\mathrm{NCM}$ & 100 \\
\hline \multirow{2}{*}{$\begin{array}{l}\text { Información } \\
\text { personal }\end{array}$} & Foto & 55 & FOT & 64 \\
\hline & $\begin{array}{l}\text { Breve descripción (Sobre quiénes son, sus hobbies, sus intereses } \\
\text { personales o profesionales, etc.) }\end{array}$ & 11 & $\mathrm{BDE}$ & 12,8 \\
\hline
\end{tabular}

Entre los aspectos sociales analizados en el blog, queda evidenciada la escasa interacción existente entre los propios alumnos, así como entre éstos y el resto de usuarios de la web, ya que son mínimas las observaciones de alguna interacción con otras webs, compartiendo enlaces de otros blogs de interés o relacionados con la temática. La apreciación de los seguidores y visitas del blog sólo ha sido posible realizarla en un pequeño porcentaje de los estudiantes, ya que la mayoría no compartían dicha información a través del complemento técnico necesario.

Finalmente, es destacable que la mayoría de ellos proporcionan una foto de perfil con la que muestran una cercanía a los lectores del blog, y, en menor medida, algunos aportan detalles sobre su vida profesional "Estudiante de Trabajo y Educación Social. Técnico Superior en Integración Social" y personal "Me Ilamo MNF y si algo me gustan son las personas. Vivo la vida intensamente y esperó seguir haciéndolo".

En suma, la herramienta, corroborando lo apuntado por Todorova, Arati, y Osburg (2010), nos ha resultado muy útil para registrar los procesos y la evaluación del aprendizaje y, en concordancia con los estudios de Aguaded, López-Meneses y Jaén (2013) hemos comprobado que pueden facilitar el conocimiento por parte del docente de los procesos de aprendizaje, la metodología de trabajo de sus clases, los procesos de tutorización y el grado de adquisición de las competencias de los estudiantes. También se puede compartir con Cebrián (2011), que favorecen una evaluación formativa en los enfoques constructivistas de la enseñanza y el aprendizaje, al ser factible un seguimiento detallado sobre la evolución de los estudiantes.

\section{CONCLUSIONES}

En este trabajo se describe una propuesta de innovación didáctica universitaria que utiliza la herramienta blog como base para la construcción de un portafolio electrónico del estudiante. De los resultados y el análisis expuesto se obtienen las siguientes conclusiones: i) Se evidencia la utilidad del portafolio electrónico para el desarrollo de nuevas habilidades y destrezas, con relación a las competencias profesionales que el estudiante ha de adquirir, ii) En particular, el trabajo ha sido útil en la construcción de competencias sobre utilización de TIC, capacidad de trabajo en equipo, emisión de juicios críticos, conocimiento y comprensión de las bases teóricas y metodológicas de la Educación Social, configuración de su campo e identidad profesional, cultural e histórica y dominio correcto de la lengua española, iii) Es posible destacar la utilización de recursos textuales y multimedia por la mayoría del alumnado, lo cual puede facilitar el desarrollo del proceso formativo desde una perspectiva constructivista, iv) En cuanto a las limitaciones, un aspecto a mejorar en posteriores experiencias sería el estímulo de una mayor interacción reflexiva-cognitiva entre el estudiantado. 


\section{REFERENCIAS}

Aguaded, J. I., López Meneses, E. y Alonso, L. Innovating with Blogs in University Courses: a Qualitative Study. The New Educational Review, 22(3-4), 103-115 (2010)

Aguaded, J. I., López Meneses, E. y Jaén, A. Portafolios electrónicos universitarios para una nueva metodología de enseñanza superior. Desarrollo de un material educativo multimedia (MEM).doi: http://dx.doi.org/10.7238/rusc.v10i1.1333 Revista de Universidad y Sociedad del Conocimiento (RUSC), 10(1), 7-28 (2013)

Alcaraz, N. La Evaluación a través de Portafolios: ¿Una Ocasión para el Aprendizaje? doi: 10.15366/riee2016.9.1 Revista Iberoamericana de Evaluación Educativa, 9 (1), 31 -46 (2016)

Alexiou, A., y Paraskeva, F. Enhancing self-regulated learning skills through the implementation of an eportfolio tool. doi: http://doi.org/10.1016/j.sbspro.2010.03.463 Procedia-Social and Behavioral Sciences, 2 (2), 3048-3054 (2010)

Amestica, L., Contreras-Higuera, W., King, A., Quezada, F. y Cornejo, E. Aprendizaje a través del uso de portfolio digital, innovando en la asignatura de formulación económica de proyectos. Revista Espacios, 37 (4), 1-5 (2016)

Arís, N. y Comas, M. À. La formación permanente en el contexto del Espacio Europeo de la Formación Permanente. doi: http://dx.doi.org/10.7238/rusc.v8i2.1049 Revista de Universidad y Sociedad del Conocimiento (RUSC), 8 (2), 5-13 (2011)

Barberà, E. La evaluación de competencias complejas: la práctica del portafolio. Educere, 31, 497-504 (2005)

Barberà, E. El estilo e-portfolio. Barcelona, UOC (2008)

Barberà, E., Gewerc, A., y Rodríguez, J. L. Portafolios electrónicos y educación superior en España: Situación y tendencias (En línea) 2009. http://www.um.es/ead/red/M8/ Acceso: 23 de Junio de 2016.

Barrett, H. C. Electronic Portfolios=Multimedia Development+Portfolio Development: The Electronic Development Process in Electronic Portfolios by American Association for Higher Education \& Accreditation, pp 110-116 AAHEA, Washington DC, USA (2001)

Barry, C. Choosing Qualitative Data Analysis Software: Atlas/ti and Nudist Compared (En línea) 2008. Recuperado de http://www.socresonline.org.uk/3/3/4.html Acceso: 04 de Marzo de 2016.

Bogdan, R. y Biklen, S. K. Investigación cualitativa de la educación. Needham Heights, MA, Allyn and Bacon (1992)

Bravo, A. A., Ramírez, G. P., Faúndez, C. A, y Astudillo, H. F. Propuesta Didáctica Constructivista para la Adquisición de Aprendizajes Significativos de Conceptos en Física de Fluidos. doi: http://dx.doi.org/10.4067/S0718-50062016000200012 Formación Universitaria, 9(2), 105-114 (2016)

Cabero, J., López-Meneses, E., y Ballesteros, C. Experiencias universitarias innovadoras con blogs para la mejora de la praxis educativa en el contexto europeo. doi: http://dx.doi.org/10.7238/rusc.v6i2.108 Revista de Universidad y Sociedad del Conocimiento (RUSC), 6 (2) (2009)

Cambridge, D. Two faces of integrative learning online. En Cambridge, D.; Cambridge, B. y Yancey, K. (Eds.): Electronic Portfolios 2.0. Emergent Research on Implementation and Impact. Virginia, Stylus (2010)

Cambridge, D. Eportfolios for Lifelong Learning and Assessment. San Francisco, Jossey-Bass (2010)

Centeno-Moreno, G., y Cubo Delgado, S. Evaluación de la competencia digital y las actitudes hacia las TIC del alumnado universitario.doi: http://dx.doi.org/10.6018/rie.31.2.169271 Revista de Investigación Educativa, 31(2), 517-536 (2013) 
Chen, H. y Light, T. Electronic portfolios and student success: Effectiveness, efficiency and learning. Washington, D.C., Association of American Colleges and Universities (2010)

Cheng, G. y Chau, J. A study of the effects of goal orientation on the reflective ability of electronic portfolio users. doi: http://dx.doi.org/10.1016/j.iheduc.2012.01.003n The Internet and Higher Education, 16, 51-56 (2012)

Cobos, D. y Vallejos, A. Implantación y desarrollo de un proyecto de utilización del portafolio electrónico por parte de alumnado universitario. Saber, Universidad de Oriente, Venezuela, 23 (1), 77-83 (2011)

Coffey, U. y Ashford-Rowe, K. The changing landscape of ePortfolios: A case study in one Australian university. Australasian Journal of Educational Technology, 30 (3), 284-294 (2014)

Darling-Hammond, L., y Snyder, J. Authentic assessment of teaching in context. doi:10.1016/S0742051X(00)00015-9Teaching and Teacher Education, 16 (5-6), 523-545 (2000)

Díaz, F., Rojas, G. Estrategias docentes para un aprendizaje significativo. México, Mc Graw Hill (1999)

EACEA/Eurydice. Modernisation of Higher Education in Europe: Funding and the Social Dimension. Brussels, EACEA P9 Eurydice (2011)

Escudero, T. Desde los tests hasta la investigación evaluativa actual. Un siglo, el XX, de intenso desarrollo de la evaluación en educación. Revista Electrónica de Investigación y Evaluación Educativa, 9 (1), (En línea) 2003. http://www.uv.es/RELIEVE/v9n1/RELIEVEv9n1_1.htm Acceso: 23 de Junio de 2016.

Estelles, S., Barbera, T. y Dema., C. Aplicación del portafolio grupal en la asignatura de Dirección de Producción y Logística: resultados de una prueba piloto. Educade, Revista de Educación en Contabilidad, Finanzas y Administración de Empresas, 4, 124-138 (2013)

Farmer, B., Yue, A., y Brooks, C. Using blogging for higher order learning in large cohort university teaching: $A$ case study.doi: http://dx.doi.org/10.14742/ajet.v24i2.1215Australasian Journal of Educational Technology,24 (2), 123-136 (2008)

Friese, S. Qualitative Data Analysis with ATLAS.ti. Newbury Park, CA, Sage (2014)

González-Sánchez, R., y García-Muiña, F. E. Recursos eficaces para el aprendizaje en entornos virtuales en el Espacio Europeo de Educación Superior: Análisis de los edublogs. Estudios sobre educación, 20, 161$180(2011)$

Hart, J. The Top 100 Tools for Learning 2011 List. Centre for Learning y Performance Technologies (En línea) 2015. http://c4lpt.co.uk/top100tools/ Acceso: 04 de Marzo de 2016.

Huffaker, D. The educated blogger: Using weblogs to promote literacy in the classroom. AACE Journal, 13 (2), 91-98 (2005)

Icarte, G. A., y Lávate, H. A. Metodología para la revisión y actualización de un diseño curricular de una carrera universitaria incorporando conceptos de aprendizaje basado en competencias. doi: http://dx.doi.org/10.4067/S0718-50062016000200002 Formación Universitaria, 9(2), 03-16 (2016)

Imbernón, F., Silva, P., y Guzmán, C. Competencias en los procesos de enseñanza-aprendizaje virtual y semipresencial. doi: http://dx.doi.org/10.3916/C36-2011-03-01 Comunicar, 36 (1), 107-114 (2011)

Jafari, A., y Kaufman, C. Handbook of Research on ePortfolios. London, Idea Group Reference (2006)

Johnson, G., y Dibiase, D. Keeping the course before the cart: Penn State's ePortfolio initiative. Educause Quarterly, 27(4), 18-26 (2004)

Johnson, R., Mims, J. S., y Doyle-Nichols, A. Developing portfolios in education: A guide to reflection, inquiry and assessment. San Francisco: Sage Publications (2006)

Joyes, G., Gray, G. y Hartnell-Young, E. Effective practice with e-portfolios: How can the UK experience inform implementation? doi: http://dx.doi.org/10.14742/ajet.v26i1.1099 Australasian Journal of Educational Technology, 26 (1), 15-27 (2010) 
Klenowski, V. Desarrollo de portafolios para el aprendizaje y la evaluación. Madrid: Narcea (2005)

Lagunes-Domínguez, A., Torres-Gastelú, C. A., Flores-García, M. A. y Rodríguez-Figueroa, A. Comparativo del uso de Tecnologías de la Información y Comunicación (TIC) por profesores de dos universidades públicas de México. doi: 10.4067/S0718-50062015000200003 Formación Universitaria, 8 (2), 11-18(2015)

López Meneses, E., Domínguez, G., Álvarez, F.J. y Jaén, A. Experiencia didáctica con estudiantes de postgrado sobre los roles del educador en la Sociedad del Conocimiento y la Comunicación con tecnologías 2.0. Revista Latinoamericana de Tecnología Educativa (RELATEC), 10 (1), 49-58 (2011)

López-Noguero, F. El análisis de contenido como método de investigación. XXI Revista de Educación, 4, 167-179 (2002)

Mansvelder, D., Beijaard, D., y Verloop, N. The portfolio as a tool for stimulating reflection by student teachers.doi:10.1016/j.tate.2006.04.033 Teaching and Teacher Education, 23(1), 47-62 (2007)

Medina Revilla, A., Domínguez Garrido, M. a C., y Sánchez Romero, C. Evaluación de las competencias de los estudiantes: modelos y técnicas para la valoración. doi: http://dx.doi.org/10.6018/rie.31.1.157601 Revista de Investigación Educativa,31(1), 239-255 (2013)

Miles, M., y Huberman, M. Qualitative data analysis: an expanded sourcebook. Newbury Park, CA: Sage (1994)

Muhr, T. ATLAS/ti - A prototype ofr the Support of Text Interpretation. Qualitative Sociology, 14(4), 349-371 (1991)

Parkes, K. A., Dredger, K. S. y Hicks, D. ePortfolio as a Measure of Reflective Practice. International Journal of ePortfolio, 3 (2), 99-115 (2013)

Parkes, K. A. y Kajder, S. Eliciting and assessing reflective practice: A case study in web 2.0 technologies. International Journal of Teaching and Learning in Higher Education, 22 (2), 218-228 (2010)

Pérez- Gómez, A. I. (Dir.). El portafolios educativo en Educación Superior. Madrid, Akal (2016)

Poyatos, C., y Allan, C. The use of learning portfolios to develop generic skills: An evaluative case study with on-line Industrial Relations students. Australian Journal of Adult Learning, 44 (1), 6-26 (2004)

Pulichino, J. Future directions in e-Learning research report. Santa Rosa, CA, USA, The eLearning Guild (2006)

Roig, R. El portafolios a través del blog: conjugando las TIC y la didáctica. En M. J. Martínez, M.J. (Coord.). El portafolios para el aprendizaje y la evaluación. Murcia, Editum (2009)

Rosa, G., Navarro-Segura, L. y López, F. El aprendizaje de las habilidades sociales en la Universidad. Análisis de una experiencia formativa en los Grados de Educación Social y Trabajo Social.doi: http://dx.doi.org/10.4067/S0718-50062014000400004 Formación Universitaria, 7 (54), 25-38 (2014)

Rosa, G., Riberas, G., Navarro-Segura, L. y Vilar, J. El coaching como herramienta de trabajo de la competencia emocional en la formación de estudiantes de Educación Social y Trabajo Social de la Universidad Ramón Llul, España.doi: http://dx.doi.org/10.4067/S0718-50062015000500009 Formación Universitaria, 8 (5), 77-90 (2015)

Saeed, N., Yamg, Y. y Sinnappan, S. Emerging Web Technologies in Higher Education: A Case of Incorporating Blogs, Podcasts and Social Bookmarks in a Web Programming Course based on Students' Learning Styles and Technology Preferences. Educational Technology and Society, 12 (4), 98-109 (2009)

Salmerón, H., Rodríguez, S., y Gutiérrez, C. Metodologías que optimizan la comunicación en entornos de aprendizaje virtual. doi:10.3916/C34-2010-03-16 Comunicar, 17 (34), 163-171 (2010)

Shang, S., Li, E.Y., Wu, Y.L. y Hou, O. Understanding web 2.0 service models: a knowledge-creating perspective. doi: 10.1016/j.im.2011.01.005 Information \& Management, 48 (4-5), 178-184 (2011) 
Shroff, R. H., Deneen, C. y Ng, E.M.W. Analysis of the technology acceptance model in examining students' behavioral intention to use an electronic portfolio system. Australasian Journal of Educational Technology, 27 (4), 600-618 (2011)

Todorova, A., Arati, D. y Osburg, T. Integrating ePortfolio in an Online Platform for Teacher Professional Development: Design and Expectations. Paper presented at the ePortfolio Conference at Learning Forum London, 5-7 Julio, London, UK (2010)

Trent, J. y Shroff, R. H. Technology, identity, and community: The role of electronic teaching portfolios in becoming a teacher. doi: http://dx.doi.org/10.1080/1475939X.2012.720416 Technology, Pedagogy and Education, 1-18 (2012)

Tubaishat, A. Can e-portfolio improve students' readiness to find an IT Career? Issues in Informing Science and Information Technology, 12, 198-202 (2015) 
